\title{
1 \\ X-ray Polarization of Gamma-Ray Bursts
}

\author{
Davide Lazzati \\ (Department of Physics, North Carolina State University, \\ Riddick Hall, Box 8202, Raleigh, NC 27695-8202)
}

\begin{abstract}
The degree and the temporal evolution of linear polarization in the prompt and afterglow emission of gamma-ray bursts is a very robust diagnostic of some key features of gamma-ray bursts jets and their micro and macro physics. In this contribution, I review the current status of the theory of polarized emission from GRB jets during the prompt, optical flash, and afterglow emission. I compare the theoretical predictions to the available observations and discuss the future prospect from both the theoretical and observational standpoints.
\end{abstract}

\subsection{Introduction}

Gamma-Ray Bursts (GRBs) are the brightest explosions in the present day Universe. Unfortunately, our understanding of their physics is still incomplete, probably due to the fact that they are short lived, point-like sources.

Polarization is a formidable tool to improve our understanding of GRB jets: their geometry, magnetization, and radiation mechanism could in principle be pinned down with a comprehensive and time-resolved analysis of linear polarization. Observationally speaking, however, polarization is not easy to measure. So far, only the optical afterglow has robust polarization measurements $(4 ; 9)$ but the diverse features and the sensitivity of the models to datail has made their interpretation, at best, controversial.

In this review, I describe the theory underlying the production of polarized radiation in GRBs in their three main phases. I will focus on X-ray polarization but the discussion will be general, since the frequency dependence of GRB polarization is very weak, especially at frequencies where Faraday rotation is not relevant. 


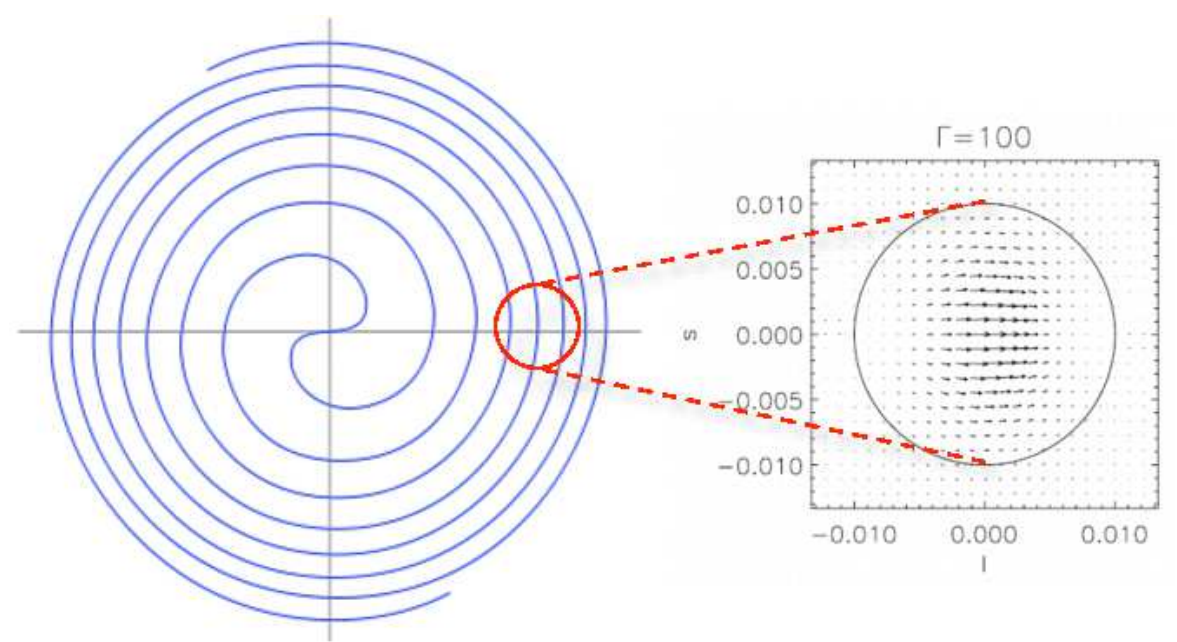

Fig. 1.1. Front view of the toroidal magnetic field in a fireball. The circle highlights the fact that, due to relativistic aberration, only a small fraction of the fireball is visible to the observer at infinite. Additional relativistic aberration effects reduce the maximum polarization, as shown in the right sub-panel (16).

\subsection{Prompt emission}

The interest in the properties of linear polarization in the prompt emission of GRBs increased dramatically with the claim that the prompt emission of GRB 021206 had a linear polarization fraction of $\sim 80 \%$ (3). Even though the claim was subsequently put into doubt $(20 ; 24)$, it generated a suite of models against which any subsequent polarization observation will be compared. In this section we present these models and compare them to observations.

- Toroidal magnetic field model - Gamma-ray burst jets are commonly believed to be produced by the effects of strong magnetization (either of a neutron star(2) or of a massive accretion disc onto a black hole(17)) combined with fast spinning. In such conditions, sufficiently far from the jet engine, the magnetic field is expected to be predominantly toroidal.

Synchrotron from a toroidal field configuration does not produce polarized radiation in normal conditions. However, due to relativistic aberration, only a small section of the whole toroidal structure produces the radiation that is detected by an observer at infinity. As a consequence, the radiation observed appears to come from a region of uniform magnetic field and is maximally polarized (see Fig. 1.1). Due to additional aber- 
ration effects, the polarization angle direction is distorted in the edges of the visible zone, slightly reducing the maximum detectable polarization (16).

Independently of details, this model predicts that almost all GRBs are strongly polarized during their prompt emission. The polarization position angle does not change with time, since the electric field vector always points towards the pole of the field, i.e., the jet axis. Only a very small fraction of bursts, those seen within an angle $1 / \Gamma$ from the jet axis, should display little to no polarization.

- $1 / \Gamma$ viewing angle effects - The main reason why most models for GRB polarization predict small values of polarization (few to ten per cent) is due to the fact that typically an observer collects radiation from different regions with different polarization orientations and the net signal is small. If, however, the fireball configuration is such that only one emission zone is observed, high polarization can be detected by the observer at infinity (23). Consider a fireball with an opening angle $\theta_{j} \sim 1 / \Gamma$ observed at an angle $\theta_{o}=1 / \Gamma$ from its edge. Due to relativistic aberration, in the comoving frame the fireball velocity and the line of sight are at a right angle. Both synchrotron radiation from a planar magnetic field $(6 ; 21 ; 8)$ and bulk inverse Compton radiation (14) are maximally polarized in that configuration.

This model can in principle account for polarization up to 100 per cent. Differently from the toroidal field model, only a small fraction of GRBs should be polarized, due to the low probability for the particular viewing configuration to be attained. As discussed for the toroidal model the electric vector points towards the jet axis and the polarization angle is therefore constant throughout the prompt emission evolution.

- Magnetic domains - If the magnetic field generated by a relativistic collisionless shock can reorganize into a uniform configuration, the fireball surface would be covered with magnetic patches, each with a different field orientation, but with a uniform field within (10). As a result of the speed of the field re-organization and of relativistic aberration, the observer at infinity sees radiation from approximately $N \sim 100$ domains. The resulting net polarization is therefore reduced by a factor $\sqrt{N} \sim 10$. This model predicts that all GRBs should be mildly polarized (in teh 10 per cent range), with rapid fluctuations of the polarization angle. The model cannot account for very high polarized fractions, as those possibly observed in the prompt emission of GRBs.

- Fragmented fireballs — The main weakness of the " $1 / \Gamma$ effects" model is that it requires a very unlikely viewing configuration. Such limitation 


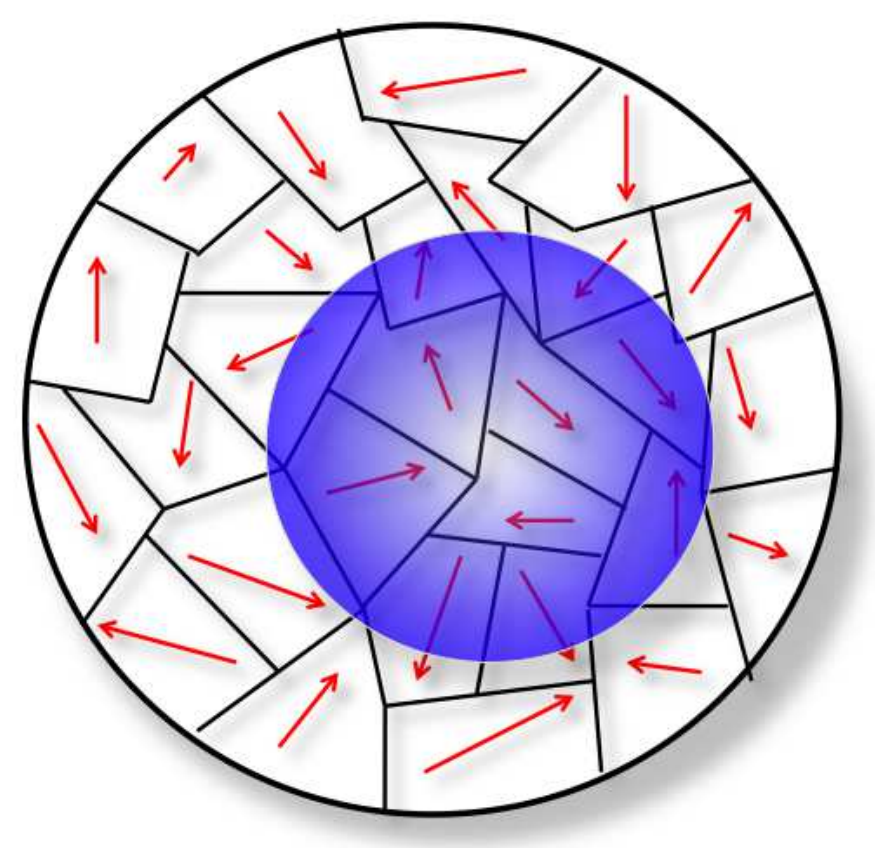

Fig. 1.2. Cartoon of the front view of a fireball with magnetic domains. The arrows show the direction of the field in the domains. The shaded circle emphasizes the fact that only some of the domains are visible by the observer.

would not be present if the fireball is fragmented in shotguns (11), cannonballs (5), or mini-jets (25). If we model a fireball as a series of identical fragments, each producing radiation with the same efficiency and moving at the same speed, polarization and intensity from a fragment are strictly correlated (15). The brightest light observed comes from the fragment that is exactly pointing at the observer. Due to cylindrical simmetry, the radiation is unpolarized. At the $1 / \Gamma$ configuration, the radiation intensity is decreased by a factor $\sim 10$, and the polarization is maximum. For viewing angles $\theta_{o}>1 / \Gamma$, both the intensity and the polarization decrease (see Fig. 1.3). Most bursts from fragmented fireballs are highly polarized if a time-resolved analysis is performed, but they are weakly polarized if the whole prompt emission is considered. That is because the electric vector points towards each fragment, and so the position angle fluctuates randomly from a pulse to the next. 


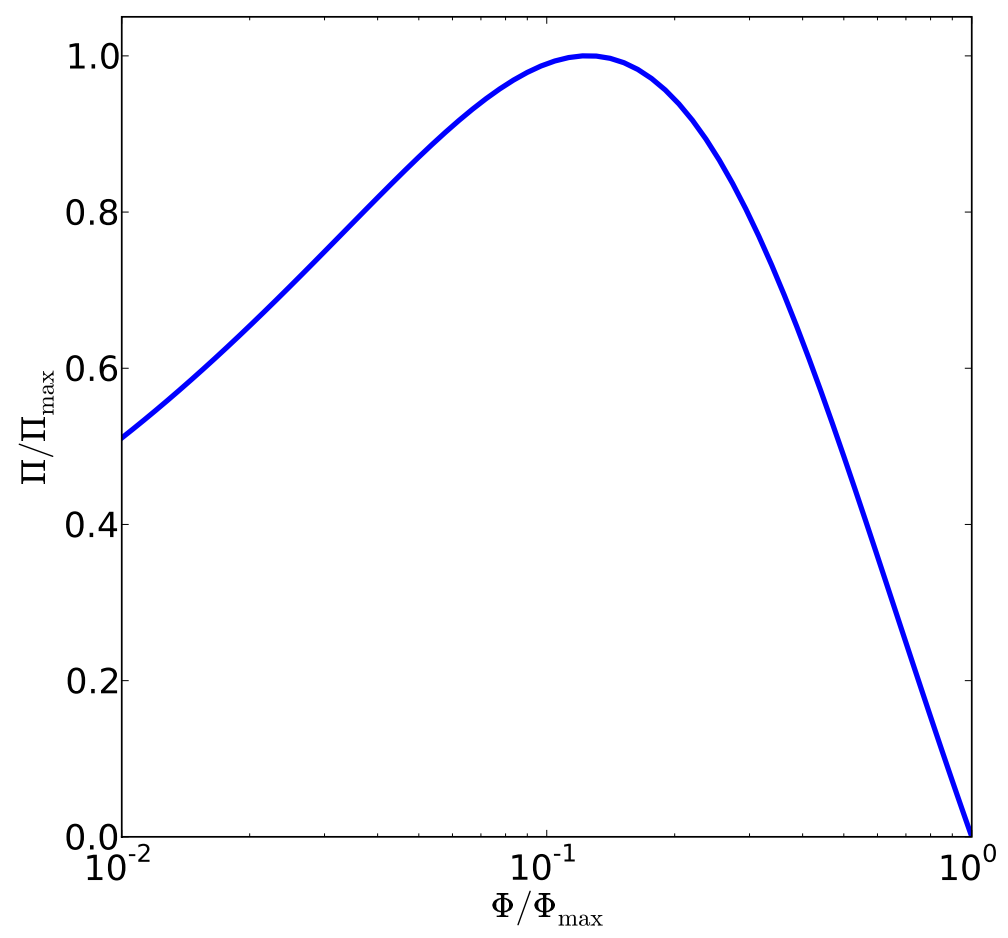

Fig. 1.3. Polarization vs. intensity for the radiation coming from a fireball made by a large number of identical fragments with negligible opening angle $\left(\theta_{\text {jet }} \ll 1 / \Gamma\right)$.

\subsection{Afterglow}

The polarization of afterglow radiation has been observed with robust results, but the comparison of observational data with models is difficult. Afterglow radiation is known to be produced by synchrotron from relativistic electrons gyrating into a shock-generated magnetic field. Detailed calculations show that the polarization from a uniform fireball is intimately connected to the evolution of the light curve $(6 ; 21)$ and is very weakly dependent on the frequency of photons (at least above optical frequencies, (19)). Initially, the polarization is vanishingly small. At times before the jet break, a small polarization of a few per cent is observed, with a position angle perpendicular to the direction towards the jet axis. At a time approximately coincident with the jet break time, the polarization vanishes again. Subsequently, it reappears, rotated by 90 degrees, reaches a maximum of $\sim 10$ per cent and eventually vanishes again (see Fig. 1.4).

The characteristic behavior of a 90 degrees rotation of the polarization 


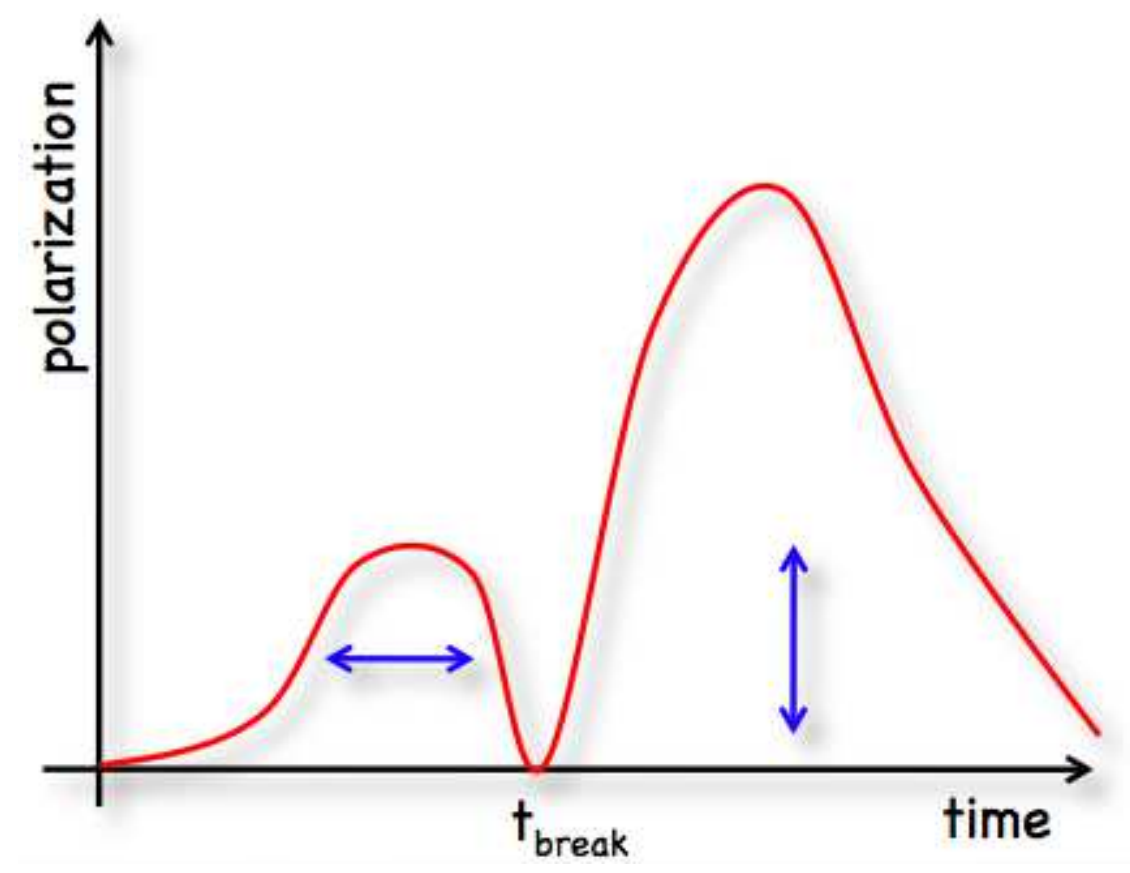

Fig. 1.4. Polarization of afterglow radiation from a uniform fireball. the arrows indicate the direction of polarization.

angle at a time roughly coincident with the jet break time is in principle a formidable prediction and was actively looked for in observations, with no success $(12 ; 13)$. It was subsequently realized that the polarization curve is very sensitive to the brightness profile of the fireball, and that fireballs with a bright core and less energetic wings produce a completely different polarization curve, with maximum polarization around the break time and a constant position angle (19). Even more complicate is the case of a fireball with bright spots randomly distributed on the emitting surface. The polarization in that case would be virtually unpredictable.

\subsection{Early afterglow}

The polarization of the prompt emission is in principle full of important information to understand the physics of GRB jets. However, polarization in the $\mathrm{X}$-ray and $\gamma$-ray regimes is hard to observe. Optical polarization is relatively easy to observe. However, models are too sensitive to details and we haven't been able to obtain much robust clues from optical polarization measurements. A potentially game changer is polarization of the early op- 
tical afterglow, also known as the optical flash. The optical flash is believed to be due to electrons in the fireball energized by the reverse shock (22). If that is the case (see (1) for alternative models) the optical flash should have the same polarization characteristic of the prompt emission (and therefore carry a lot of insight) combined with the same ease of observation of the afterglow polarization (13).

\subsection{Discussion}

After discussing the polarization of the various stages of GRB emission in detail, we here compare them with each other and with observations and focus more on the X-ray aspects and future perspectives. Prompt emission polarization is certainly the most appealing from the theoretical point of view. Models are able to deliver univocal interpretation for the various observational scenarios. The few available observations are, however, inconclusive and contradictory. Early observations claimed a high polarization for the overall burst (3). More recent observations find, instead, that the polarization is indeed large, but the position angle varies from pulse to pulse (7). The observations are different and so too are the implications. Constant position angle and high polarization point to a toroidal magnetic field model, while variable angle is indicative of a fragmented fireball scenario. The perspective of an early afterglow polarization measurement is exciting, but the optical flash has no emission in the X-rays, and its theoretical interpretation is still a matter of open debate. While a positive measurement of large polarization would be interesting, a no-polarization result, as the one for GRB 060418 (18), would be open to very many interpretations. Afterglow observations are plagued by the model sensitivity to details, with the notable exception of polarization of the X-ray flares (Fan, this volume) which are supposed to be due to engine activity and could therefore be polarized in the same way the prompt emission is.

At the end of the day, what would be the best choice for an X-ray polarimeter? Disregarding technical challenges, a theoretician would try to observe prompt emission first, X-ray flashes second, and the afterglow emission only as a last resort.

\section{References}

[1] Beloborodov, A. M. (2002). ApJ 565, 808-828.

[2] Bucciantini, N., Quataert, E., Arons, J., Metzger, B. D. and Thompson, T. A. (2008). MNRAS 383, L25-L29.

[3] Coburn, W. and Boggs, S. E. (2003). Nature 423, 415-417. 
[4] Covino, S. et al. (1999). A $\mathcal{E} A$ 348, L1-L4.

[5] Dado, S., Dar, A. (2009). arXiv:0901.4260

[6] Ghisellini, G., Lazzati, D. (1999). MNRAS 309, L7-L11.

[7] Götz, D., Laurent, P., Lebrun, F., Daigne, F., Bošnjak, Ž. (2009). ApJ 695, L208-L212.

[8] Granot, J. (2003). ApJ 596, L17-L21.

[9] Greiner, J. et al. (2003). Nature 426, 157-159.

[10] Gruzinov, A., Waxman, E. (1999). ApJ 511, 852-861.

[11] Heinz, S., Begelman, M. C. (1999). ApJ 527, L35-L38.

[12] Lazzati, D. et al. (2003). A $\mathscr{S} A$ 410, 823-831.

[13] Lazzati, D. et al. (2004). A\&A 422, 121-128.

[14] Lazzati, D., Rossi, E. M., Ghisellini, G., Rees, M. J. (2004). MNRAS 347, L1-L5.

[15] Lazzati, D., Begelman, M. C. (2009). Submitted to ApJL.

[16] Lyutikov, M., Pariev, V. I., Blandford, R. D. (2003). ApJ, 597, 998-1009.

[17] MacFadyen, A. I., Woosley, S. E. (1999). ApJ 524, 262-289.

[18] Mundell, C. G. et al. (2007). Science 315, 1822.

[19] Rossi, E. M., Lazzati, D., Salmonson, J., Ghisellini, G. (2004). MNRAS 354, 86-100.

[20] Rutledge, R. E. and Fox, D. B. (2004). MNRAS 350, 1288-1300.

[21] Sari, R. (1999). ApJ 524, L43-L46.

[22] Sari, R., Piran, T. (1999). ApJ 520, 641-649.

[23] Waxman, E. (2003). Nature 423, 388-389.

[24] Wigger, C., Hajdas, W., Arzner, K., Güdel, M. and Zehnder, A. (2004). ApJ 613, 1088-1100.

[25] Yamazaki, R., Ioka, K., Nakamura, T., Toma, K. (2006). Advances in Space Research 38, 1299-1302. 